\title{
Une flûte de Pan peu bucolique
}

\section{Marianne Mesnil}

\section{OpenEdition}

\section{Journals}

Édition électronique

URL : http://journals.openedition.org/ethnomusicologie/2377

ISSN : 2235-7688

Éditeur

ADEM - Ateliers d'ethnomusicologie

Édition imprimée

Date de publication : 1 janvier 1990

Pagination : $35-43$

ISBN : 2-8257-0423-7

ISSN : $1662-372 X$

Référence électronique

Marianne Mesnil, « Une flûte de Pan peu bucolique », Cahiers d'ethnomusicologie [En ligne], 3 | 1990,

mis en ligne le 15 octobre 2011, consulté le 21 avril 2019. URL : http://journals.openedition.org/ ethnomusicologie/2377 


\title{
UNE FLÛTE DE PAN PEU BUCOLIQUE
}

\author{
Marianne Mesnil
}

\section{Note d'actualisation}

Cet article a été rédigé avant que ne soit largement répercutée la nouvelle «ligne idéologique» de Ceaucescu et bien sûr, avant les derniers événements de décembre 1989 qui y mirent un point final. Dès lors, il est peut-être utile de situer les propos qui suivent par rapport à une situation plus récente que celle à laquelle se réfère l'article.

En effet, le phénomène Zamfir, comme j'ai tenté de le montrer dans ces lignes, est l'expression d'une époque précise où furent réunies les doubles conditions d'une rencontre Est-Ouest, et plus particulièrement d'une rencontre entre la Roumanie et la France. Cette période correspond à ce que l'on pourrait appeler «les années d'illusion» du régime Ceaucescu durant lesquelles le grand public a pu croire (même en Roumanie, jusqu'à un certain point), à une déstalinisation du régime et à une véritable ouverture politique et culturelle.

Zamfir et sa flûte de Pan furent bien les ambassadeurs de cette période. L'une des valeurs idéologiques dont le régime se fit alors le défenseur, au moins dans son discours sinon dans sa politique, fut le nationalisme traditionnaliste et paysan. Le «folklore» fut donc mobilisé pour exalter l'ancienneté et la spécificité de la culture roumaine, et pour renforcer la politique nationaliste contre les «frères ennemis», slaves et hongrois en particulier.

Mais vers les années quatre-vingt, une rupture s'est produite par rapport à ce type de position officielle. Elle se manifeste par l'apparition de la notion d'homme nouveau: c'est lui que le Conducator lance précisément à l'assaut de la tradition et de la continuité paysanne qu'il avait mobilisées jusque-là.

C'est avec l'idéologie de l'homme nouveau que se dessine à l'horizon de la Roumanie le paysage des «agro-villes» auxquelles les villages traditionnels devront faire place. La ligne officielle requiert dès lors que toute référence aux racines du passé rural soit effacée pour permettre à l'idéologie nouvelle de 
triompher. Du point de vue folklorique, c'est l'ère de Cîntarea României" qui s'ouvre, dernier clin d'œil aux références du XIX ${ }^{\mathrm{e}}$ siècle: mais on y chantera de moins en moins l'âme du peuple, la nature ou le dor (nostalgie) de la maison. Cette vaste opération médiatique, que l'on pourrait comparer à une sorte d'Eurovision en vase clos à la gloire exclusive du Génie des Carpathes, ne laissera plus place à d'autres expressions populaires qu'à ce folklore officiel, exclusivement orienté vers le «culte de la personnalité» du numéro un roumain.

On comprend dès lors que, dans une telle réorientation du discours Ceaucescu, Zamfir et sa flûte de Pan n'aient plus eu de rôle à jouer, quelle qu'ait été l'évolution politique personnelle du musicien. Dans ce nouveau contexte, il n'y avait place pour d'autres vedettes que pour des chantres très officiels attachés à la personne du tyran: ce fut sans doute le rôle qu'occupa un homme comme le poète Andrian Paunescu (que les images d'actualité nous ont montré au moment où il réussissait à se réfugier à l'ambassade des États-Unis). Mais on comprend pourquoi ces nouvelles vedettes ne furent guère exportables en Occident!

M. M., janvier 1990

L'évocation de la musique populaire roumaine suscite inévitablement des accents de flûte de Pan et, sans doute, la virtuosité d'un Gheorghe Zamfir a-telle contribué à renforcer ce stéréotype culturel devenu image de marque international de la "roumanité».

Et pourtant, il serait difficile de trouver, de nos jours, dans le riche éventail des productions musicales de la paysannerie roumaine, manifestation moins bucolique que celle de cet instrument.

Pourquoi, dès lors, cette «flûte à vingt tuyaux» est-elle devenue le symbole musical de ce folklore?

La question n'est pas anodine, car le phénomène tient à une rencontre historique déterminée: celle de l'Occident des Golden Sixties (et Paris en particulier) qui tourne à nouveau son regard vers «l'autre Europe», au moment où celle-ci opère son ouverture sur l'Occident. Ceci est particulièrement vrai pour la Roumanie qui, après la mort de Gheorghiu-Dej (1965), sort de son engourdissement stalinien (malheureusement pour peu de temps puisque les retombées de Prague seront bientôt prétexte à favoriser une nouvelle régression sociale).

Gheorghe Zamfir se fait donc le chantre de la Roumanie renaissante des années 1965 à 1970, comme d'autres s'étaient faits les chantres de la Roumanie à naître des années 1848 .

Mais les temps changent et leurs ambassadeurs culturels sont à leur mesure: les Vasile Alexandri et autres intellectuels découvreurs du «génie des

\footnotetext{
L'expression Cîntarea României (le chant de la Roumanie) est empruntée au titre d'un poème en prose attribué à la plume de Alecu Russo, l'un des écrivains de la génération des révolutionnaires de 1848. Le poème fut publié par Vasile Alexandri dans la revue Romania Literara.
} 
peuples» avaient su émouvoir les porte-parole des idées de la France progressiste de l'époque, en traduisant les premiers recueils de "poésie populaire» qui surent rencontrer le goût du Romantisme révolutionnaire. Ils servaient ainsi la cause de cette intelligentsia émigrée qui cherchait des alliés auprès des grandes puissances occidentales pour conquérir leur indépendance nationale ${ }^{1}$.

Si la poésie populaire et plus particulièrement la doina d'Alexandri ${ }^{2}$ fut alors mise au service des revendications de ce «printemps des peuples», c'est elle que nous retrouvons comme favorite du répertoire de Zamfir, à nouveau mobilisée pour émouvoir et conquérir, non plus une élite intellectuelle, mais un public saturé de musique pop par les mass media et à la recherche d'un «ressourcement» musical. Bien que le public soit trompé sur la marchandise qu'on lui vend et que le vendeur soit lui-même abusé par son propre produit, conviction et ferveur seront de la partie et chacun y trouvera son compte.

Pour comprendre ce qui précède, il faut se souvenir que lorsque Zamfir débarque en Occident, d'autres virtuoses de la flûte de Pan ont déjà préparé le terrain. A l'exposition universelle de Paris déjà (1889), le public découvrait la flûte de Pan sous les doigts d'un Angeluş Dinicu. Puis, dans l'entre-deuxguerres, c'est la figure de Fanica Luca, également célèbre comme chanteur de romances, qui s'impose lors de tournées internationales. C'est encore lui qui devient chef de file d'une école de flûte de Pan créée à l'initiative du nouveau régime, et qui reprend la vieille tradition des ménétriers tsiganes professionnels. De cet enseignement sortiront des interprètes comme Radu Simion ou Simion Stanciu qui appartiennent à la même génération que Zamfir et se tailleront aussi un petit succès international.

Pourquoi, dès lors, ce «phénomène Zamfir»? Le vedettariat a ses lois, mais cela n'explique pas tout.

Je risque ici une hypothèse: parmi ces virtuoses d'un instrument dont le nom antique n'est pas pour déplaire à notre recherche d'archaïsme, il s'en est trouvé un, mieux placé pour suggérer l'idée de continuité de la culture traditionnelle roumaine depuis l'origine de son peuple. Seul Zamfir offrait à la fois ces qualités de virtuose, et un profil de «vrai» Roumain compatible avec l'image du «berger des Carpathes »: car Zamfir fait figure d'exception au sein d'une autre tradition, celle des ménétriers tsiganes dont on connaît l'importance dans la vie musicale de cette partie de l'Europe. Le rôle de ces lăutari est d'ailleurs complexe, puisqu'ils sont parfois les garants d'une certaine mémoire collective, là où la paysannerie abandonne ses propres traditions musicales. C'est le cas de certaines régions comme l'Olténie où les petites formations de musiciens paysans qui se produisaient lors de circonstances telles que noces ou fêtes villageoises, ont rapidement cédé la place à ces ménétriers tsiganes qui ont appris le

Voir Alexandri (1982) et Mesnil (1983).

2 V. Alexandri (1982:223, note) définit les doine (pl. de doina, n.d.l.r.) comme «des chants d'amour, de tristesse et de nostalgie (dor), des pleurs mélancoliques de l'âme roumaine dans toutes les circonstances de sa vie». 


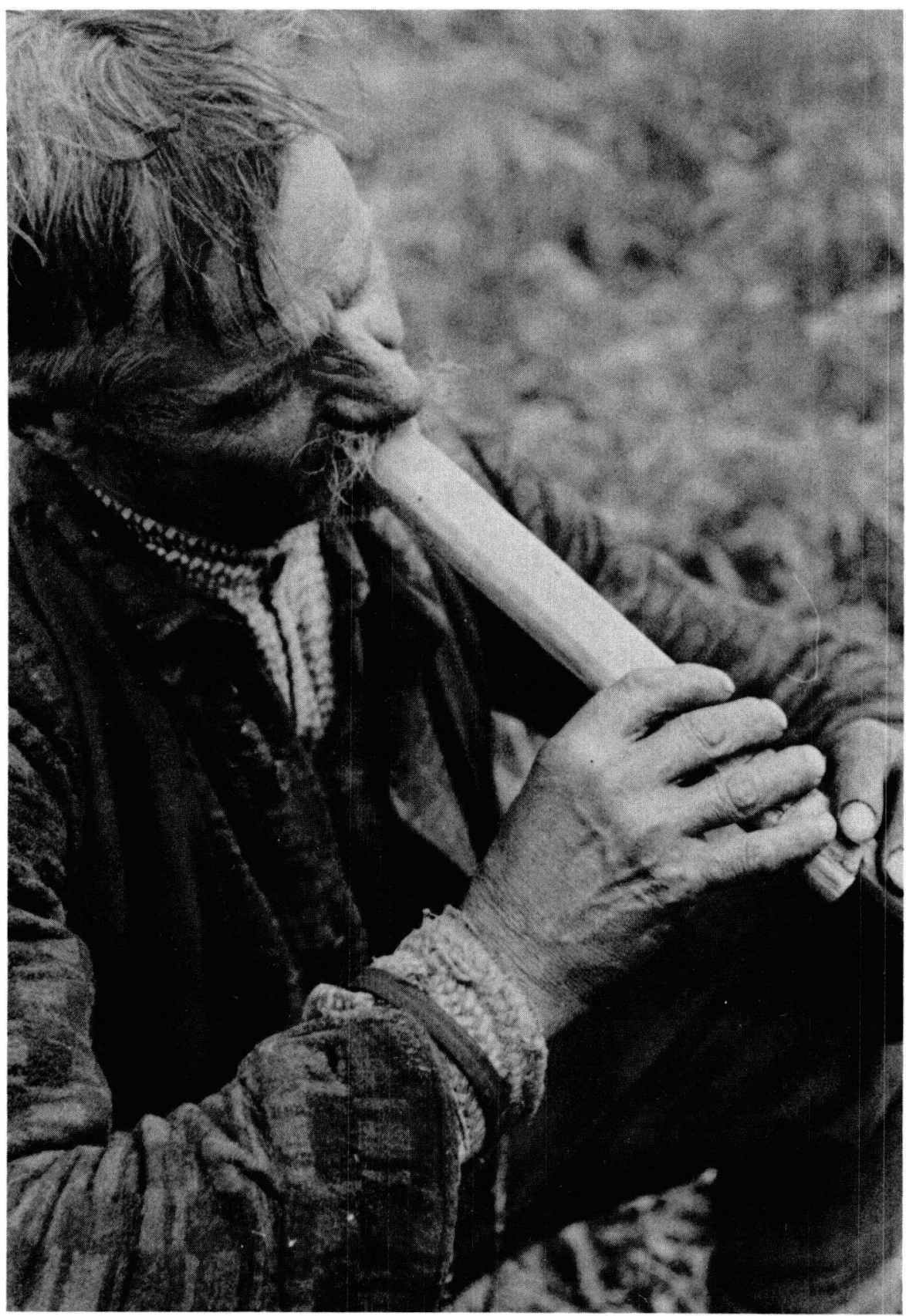

Fig. 1: Le vieux «Tsantsa» est spécialiste dans l'interprétation du rôle de l'Ours des mascarades de Nouvel An. Il interprète ici une doina sur sa flûte. Village de Vîrful Cîmpului (Moldavie du Nord), juin 1970. (Photo: M. Mesnil). 
répertoire souhaité pour ces fêtes. Ces «professionnels» au service de la paysannerie ont ainsi pris en charge une tradition musicale locale ou régionale qui va resurgir grâce à eux dans le folklore urbain, tout en exerçant à son tour une influence urbaine sur la paysannerie. En effet, l'autre aspect du rôle joué par ces professionnels de la tradition musicale orale concerne précisément ce folkore urbain que nous avons trop souvent confondu avec la musique «tsigane» ${ }^{3}$. Ce sont les mêmes lăutari que nous retrouvons dans les évocations «couleur locale» des villes de l'avant- et de l'entre-deux-guerres; de bistrot en restaurant et de table en table, ils animent les soirées en chantant leurs rengaines selon le goût du client. Et le «client», c'est ce monde de contraste, fait de paysannerie mal dégrossie et d'urbanisme mal enraciné; de boyards venus s'égarer un soir dans les mahale 4 pour oublier que la page de leur histoire est en train d'être tournée; un monde où se rencontrent des regards encore embués dans les fastes de l'Orient et déjà chargés des rêves de modernité.

C'est la mosaïque humaine qu'évoque P. Morand dans son Bucarest au pittoresque parfois douteux, ou encore le monde déjà nostalgique d'un Matei Caragiale 5 .

Mais revenons-en à notre chantre de la «roumanité» des années 1965 à 1970. Il est lui aussi le produit d'une formation musicale urbaine, puisqu'il commence ses études dans un «lycée de musique» à Bucarest et poursuit sa formation au Conservatoire Ciprian Porumbescu, également à Bucarest. Et de fait, c'est à la ville que l'on peut encore apprendre l'art de cet instrument, car il y a longtemps déjà que les Carpathes ont cessé de se faire l'écho des plaintes de la syrinx dont, soit dit en passant, le nom roumain de nai n'indique aucune filiation avec la mythologie antique, contrairement à son équivalent français ou anglais (Panpipe).

Gheorghe Zamfir va alors permettre de joindre deux termes contradictoires, afin d'effacer leur incompatibilité historique au profit d'une illusion de continuité culturelle dont la Roumanie officielle et la petite bourgeoisie postrévolutionnaire qui s'en fait le relais, tenteront de multiplier les effets. Formé à l'école urbaine grâce à une tradition largement prise en charge par les Tsiganes

3 Il s'agit d'une musique jouée par les orchestres tsiganes, mais qui est en fait, selon l'expression de Béla Bartók, «une musique savante à caractère populaire». A Budapest, où ce type de musique s'est particulièrement développé, elle est connue sous le nom de «Magyar notak». Sur l'influence mutuelle des folklores musicaux paysan et urbain, voir par exemple G. Ciobanu (1979).

4 Mahale est le nom qui désigne les faubourgs dont l'ambiance balkanique n'a pas manqué de fournir une source d'inspiration à la littérature.

C'est le poète populaire Anton Pann, né à la fin du XVIII ${ }^{e}$ siècle, qui s'est fait le premier porteparole de ce folklore urbain; on lui doit l'expression de «chant de mahala» (cîntec de mahala) qui désigne ce folklore musical influencé par l'Orient, et dont les faubourgs de Bucarest semblent avoir été un lieu privilégié de manifestation.

5 Cf. P. Morand (1935). Quelques pages de son «album pittoresque» sont notamment consacrées à cette mahala. La mahala, dit-il, «c'est le royaume des ruines, c'est l'Orient qui n'enterre pas ses cadavres, qui ne balaie pas ses ordures. C'est le fatalisme appliqué à la destinée des maisons comme à celle des hommes [...]» (p. 135). Voir aussi Matei Caragiale (1929). 


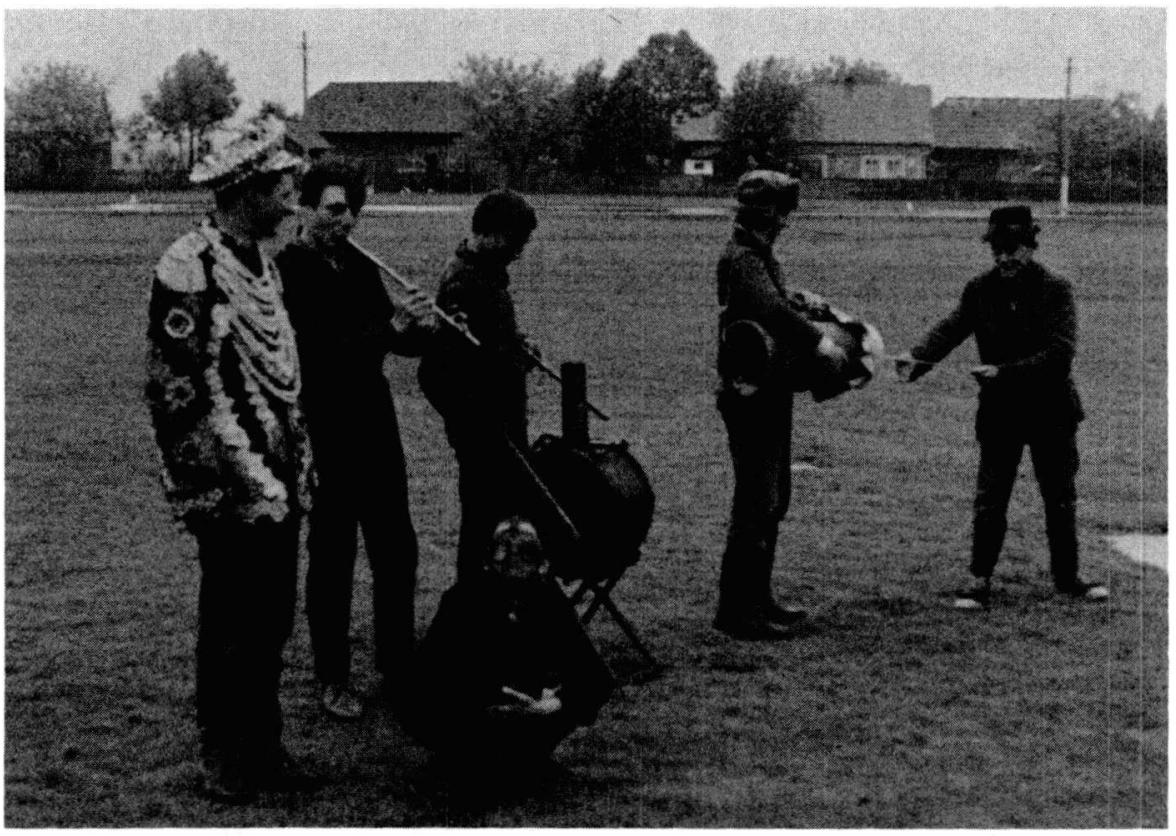

Fig. 2: Un groupe de jeunes ont sorti pour nous leurs instruments de musique qui accompagnent la troupe d'interprètes de théâtre populaire du Nouvel An. On y distingue une flûte longue, un tambour (toba) sur trépied et un buhai (littéralement «bœuf»), instrument exclusivement rituel qui accompagne les souhaits de fertilité du cycle des Douze jours. A l'avant-plan, un jeune homme en costume de théâtre et, accroupi, un autre portant un «masque à gaz», récupération de guerre, qui entre parfois dans ces travestis du Nouvel An. Village de Badauti (Moldavie du Nord), juin 1970. (Photo: M. Mesnil).

lăutari, «Zamfir le Roumain» devient l'incarnation de cette continuité pour l'Occident, mais aussi pour une nouvelle classe sociale à la recherche de son identité nationale ${ }^{6}$.

Ainsi donc se trouvent occultées les ruptures que la tradition orale a connues au cours de deux longs millénaires d'une histoire complexe, entre la plainte du vieux dieu arcadien et celle du «berger des Carpathes», garant de l'antériorité du peuplement roumain sur tout autre mouvement de migration (argument d'autorité contre d'éventuelles revendications territoriales). Cette continuité est soulignée avec insistance dans la plupart des textes de présentation qui figurent sur les pochettes des disques de flûte de Pan: on y évoque la légende antique de la nymphe Syrinx transformée en roseau pour échapper à Pan et avec laquelle le dieu confectionne la première flûte qui portera son nom et perpétuera sa plainte.

\footnotetext{
6 Voir à ce propos Karnoouh 1984.
} 
L'effet de sens «Zamfir» se précise: d'une part, il permet d'affirmer les valeurs d'identité nationale de la Roumanie de 1965, à la fois à l'usage de la nouvelle culture de masse et de l'Occident, prêt à être séduit. Et d'autre part, il sert à occulter la rupture entre une pratique musicale antique et le développement d'une technique virtuose par la tradition des Tsiganes lăutari qui constitue elle-même le réceptacle de courants culturels multiples.

Enfin, les prestations de Zamfir proposent une réponse parfaitement adéquate à la demande occidentale du moment, car elles fournissent un exotisme musical qui a tout pour plaire, étant pris pour ce qu'il n'est pas: les derniers accents authentiques d'une culture bucolique qui nous vient du fond des âges et qui purifie notre oreille saturée de la trop bruyante guitare électrique. En témoigne le morceau de lyrisme qu'adresse Marcel Cellier sous forme d'une lettre ouverte à Electrecord, reproduite sur la pochette d'un disque de Zamfir en version bilingue (français-anglais) ${ }^{7}$. Je ne résiste pas à en recopier ici quelques lignes:

"A force de traîner dans toutes les coulisses de tous les music-hall, d'y ingurgiter du pire et du meilleur et de naviguer dans le no man's land de succédané de Beatles et de souvent maladroits pilleurs de Folklore et de Classique, j'avais fini par perdre mon émotivité [...]. Et brutalement, avec les cîntece bătrineşti, Paris s'asseyait à la table des noces paysannes, avec une hora, Paris entrait dans la ronde [...] et après s'être laissé aller, pour reprendre son souffle, à la mélancolique rêverie d'une doina, Paris se lançait dans une impossible sîrba pour finalement se déchaîner dans un obsédant căluş. Paris était ces lăutari et ces căluşari, ces bergers et ces paysans [... ${ }^{8}$ ».

Comme on le voit, la méprise est totale, et le succès à sa mesure. Elle est d'autant plus aisée qu'entretemps, le répertoire a évolué, car avec Zamfir, on abandonne le côté «mahala» et ses accents de nostalgie orientale, au profit d'un répertoire tiré de la musique paysanne roumaine, mais complètement désincarnée: les airs qui accompagnent les moments forts de la vie du village (mariage, hora (ronde), sortie de masques) sont subitement projetés sur la scène des cabarets et music-hall d'Occident, et traduits en de nouvelles orchestrations dont la vedette est tenue par cette flûte oubliée des bergers, le nai.

7 Cf. disque Electrecord L'extraordinaire flûte de Pan de Gheorghe Zamfir, collection «Trésors folkloriques roumains», s.d.

8 Les cintece batrîneşti (littéralement «chants ancestraux») et les hore (pluriel de hora, «ronde paysanne») sont avec les doine les trois catégories retenues par V. Alexandri (op. cit.) pour désigner la poésie populaire. Il définit les cintece batrîneşti ou «ballades» comme de petits poèmes traitant d'événements historiques et de hauts-faits. Les hore sont des chants de joie du peuple (cf. op.cit., p. 33). Ces manifestations poétiques correspondent à des chants ou danses populaires.

La sîrba est également une danse tout comme le caluş (ou caluşar), célèbre par le caractère acrobatique de ses figures. Sur les danses populaires roumaines, deux ouvrages ont paru récemment: Bucşan (1971) et Birlea (1982). 
On n'en ravive pas moins l'antique légende, ignorant du même coup qu'au sein de cette paysannerie donnée en spectacle sous une forme qui ne lui appartient pas circulent d'autres récits qui racontent également des histoires de flûtes enchantées.

C'est le cas par exemple d'un conte de Transylvanie publié en 1895 par D. Stancescu ${ }^{9}$ : «Le jeune homme et l'aigle». Il y est question de deux jeunes filles qui, pour échapper, l'une à un mariage incestueux, l'autre aux poursuites de sa marâtre, sont changées en roseaux au milieu d'un lac. Mais un berger survient qui coupe les deux roseaux pour s'en faire deux pipeaux. Et lorsqu'il veut en jouer, chaque flûte se met à parler par la voix des jeunes filles qui prient le berger de les mener chez l'Empereur...

Ce thème du «roseau plaintif» est répandu à travers toute l'Europe ${ }^{10}$; l'histoire de la syrinx antique n'est donc qu'une variante parmi d'autres, d'un thème qui n'a cessé d'inspirer la tradition orale. Cela nous indique qu'une telle tradition n'est pas dépourvue de toute continuité; mais encore faut-il la chercher là où elle se manifeste réellement.

Quant à ces joueurs de pipeau, ils ne sont pas des créations mythiques; et leur répertoire a certes contribué à enrichir le trésor musical patiemment recueilli par des chercheurs qui ont consacré leur vie à l'étude systématique de ce type de traditions. Les travaux d'un Béla Bartók ou d'un Constantin Brăiloiu ${ }^{11}$ sont autant de jalons qui nous guident aujourd'hui sur le chemin de la connaissance de ces cultures orales en voie d'extinction.

Cf. «Flacaul si pajura» in Stancescu (1895).

10 Voir par exemple le thème d'une romance portugaise «Peregrina» in Puymaigre (1881).

11 Les œuvres de l'ethnomusicologue roumain C. Brăiloiu ont été récemment republiées chez Editura Muzicale à Bucarest par les soins d'E. Comişel, édition partiellement bilingue (roumain-français). Le volume $\mathrm{V}$ a paru en 1981. 


\section{Bibliographie}

\section{ALEXANDRI Vasile}

1866 Poezi popolare ale românilor. Bucarest. [Traduction française: Bucarest: Minerva, 1982].

BIRLEA O.

1982 Eseu despre dansul popular romanesc. Bucarest: Carta Romaneasce.

BUCŞAN A.

1971 Specificul dansului popular romanesc. Bucarest.

CARAGIALE Matei

1929 Crai de curtea Veche. Bucarest. [Traduction française: Les seigneurs du vieux castel. Lausanne: L'Age d'Homme, 1969].

KARNOOUH Claude

1984 «Une genèse allégorique du politique; le folklore». Paris. [ronéo].

MESNIL Marianne

1983 «Entre nationalisme et romantisme: naissance d'une discipline», Revue de l'Institut de sociologie, Bruxelles, 3-4: 455-66.

MORAND Paul

1935 Bucarest. Paris: Plon.

PUYMAIGRE Comte de

1881 Romanceiro (Choix de vieux chants portugais...), Paris: Ernest Leroux.

\section{STANCESCU D.}

1895 «Flacaul şi pajura», in: Glume şi poveşti. Republié in: Niscov V., cele Trei Rodii aurite, Bucarest: Minerva, 1979. [ «Le jeune homme et l'aigle» in Farces et contes, republié in Les trois fruits d'or]. 